\title{
Distribution et écologie des Méduses (Cnidaria) du golfe de Tunis (Méditerranée sud occidentale)
}

\section{Distribution and ecology of Medusae and Scyphomedusae (Cnidaria) in Tunis Gulf (SW Mediterranean)}

\author{
M.N. Daly Yahia ${ }^{\mathrm{a}, *}, \mathrm{~J}$. Goy ${ }^{\mathrm{b}}$, O. Daly Yahia-Kéfi ${ }^{\mathrm{c}}$ \\ ${ }^{a}$ Laboratoire de biosurveillance de l'environnement, Groupe de recherche en hydrologie et en planctonologie, \\ Faculté des sciences de Bizerte, 7021 Zarzouna, Tunisia \\ ${ }^{b}$ Institut océanographique, 195, rue Saint-Jacques, 75005 Paris France. \\ ${ }^{c}$ Laboratoire de planctonologie, Institut national agronomique de Tunisie, 43, avenue Charles- Nicolle, 1082 Tunis, Tunisia
}

Reçu le 26 décembre 2002 ; révisé et accepté le 30 mai 2003

\begin{abstract}
Résumé
Les méduses du golfe de Tunis représentées par 25 espèces constituent du point de vue taxonomique le troisième groupe zooplanctonique après les Tintinnides et les Copépodes. Parmi les 20 Hydroméduses recensées au cours du cycle d'étude (décembre 1993-novembre 1995), les anthoméduses (huit espèces), les trachyméduses (cinq espèces) et les leptoméduses (quatre espèces) sont les plus diversifiées. Les Limnoméduses (deux espèces) et les Narcoméduses (une espèce) étant les sous-classes les moins représentées. Ce sont les espèces du genre Obelia et Clytia qui commandent la dynamique quantitative des hydroméduses dans la baie avec des poussées susceptibles d'atteindre des valeurs exceptionnelles de l'ordre de $25800 \mathrm{ind} / \mathrm{m}^{3}$ pour Obelia spp. Au sein des scyphoméduses dont la richesse spécifique s'élève à cinq espèces, Pelagia noctiluca est l'espèce la plus commune, dont les invasions s'observent en saison automnale et surtout hivernale. Le golfe de Tunis apparaît comme formé par deux types de communautés de Méduses ; la première endogène constituée d'espèces constantes dans leur apparition tel que Olindias, Cladonema, Aurelia, Cotylorhiza et Rhizostoma et parfois pérennes comme Obelia spp., Clytia spp.; la deuxième exogène à répartition spatiotemporelle irrégulière et représentée par des espèces visiteuses comme Velella, Sminthea ou encore Pelagia dont la présence sur la côte résulte de la présence de vents et de courants locaux particuliers.
\end{abstract}

(C) 2003 Publié par Éditions scientifiques et médicales Elsevier SAS.

\begin{abstract}
The Medusae of Tunis Gulf ( 25 species) are qualitatively the third zooplanktonic group after Tintinnids and Copepods. Anthomedusae (eight species), Trachymedusae (five species) and Leptomedusae (four species) represent the major part among the 20 species of Hydromedusae identified during our study (December 1993-November 1995). However, the Limnomedusae (two species) and the Narcomedusae (one species) are poorly represented. In the Bay of Tunis, Obelia spp., the most common and dominant species, command the quantitative fluctuations of Hydromedusae and reach exceptionally the density of 25800 ind. $\mathrm{m}^{-3}$. Within the Scyphomedusae (five species), Pelagia noctiluca is the most common species which outbreaks in winter and autumn. Tunis Gulf seems to be composed of two jellyfish communities; the first one is composed of endogenous species showing a constant period of blooming like Olindias, Cladonema, Aurelia, Cotylorhiza and Rhizostoma and sometimes permanent like Obelia spp. and Clytia spp.; the second is represented by exogenous species considered as "visitors" like Velella, Sminthea or Pelagia whose presence on the coast is dependent on local and particular winds and currents.
\end{abstract}

() 2003 Publié par Éditions scientifiques et médicales Elsevier SAS.

Mots clés : Méduses ; Écologie ; Golfe de Tunis ; Méditerranée sud-occidentale

Keywords: Jellyfish; Ecology; Tunis Gulf; SW Mediterranean

* Corresponding author.

E-mail address: dalyyaha.ons@inat.agrinet.tn (M.N. Daly Yahia). 


\section{Introduction}

Dans le cadre de recherches sur l'ensemble des organismes planctoniques effectuées dans la baie de Tunis (décembre 1993-novembre 1995) et dans le golfe de Tunis (novembre 1995), les méduses se sont révélées un groupe intéressant à étudier d'une manière approfondie car il existe peu d'informations sur la rive méridionale de la Méditerranée. En effet, la plupart des inventaires faunistiques réalisés dans le cadre des observations faites par les stations marines, en particulier les stations de Naples (Brinckmann-Voss, 1970, 1987), Villefranche-sur-Mer (Trégouboff and Rose, 1957 ; Goy, 1972 ; Matsakis, 1990 ; Buecher, 1996 ; Goy, 1997), Marseille (Berhaut, 1969), Banyuls (Razouls and Thiriot, 1968) et Barcelone (Vives, 1966 ; Gili et al., 1987) ont incité des travaux parfois dès le début de leur création.

En Tunisie, les seules études sont ponctuelles et souvent d'ordre qualitatif : Séguin (1968) a recensé sur le littoral nord tunisien trois Hydroméduses (Rhopalonema velatum, Aglaura hemistoma et Solmundella bitentaculata), tandis que Chakroun et Alaoui-Bejaoui (1995) n'ont recensé que deux Scyphoméduses Aurelia aurita et Pelagia noctiluca.

De plus, le rôle de ces organismes du plancton gélatineux à la base de la chaîne alimentaire marine hétérotrophique est aujourd'hui largement prouvé et les travaux de la CIESM
(2001) montrent l'intérêt de l'étude des Méduses en particulier et surtout leurs impacts sur le fonctionnement de l'écosystème pélagique, sur certaines activités humaines comme le tourisme, la pêche et diverses activités industrielles et sur la santé humaine.

Ce travail se propose de répondre à un double objectif scientifique. D'abord réaliser le premier inventaire taxonomique des Méduses du golfe de Tunis en tenant compte de leur écologie et du calendrier d'apparition des principales sous-classes et espèces, ainsi que leur distribution spatiotemporelle sur un cycle de deux années d'étude. Ensuite, essayer de montrer comment s'effectuent les échanges entre le golfe et la baie de Tunis quant aux populations d'Hydroméduses et de Scyphoméduses.

\section{Matériel et méthodes}

\subsection{Situation géographique (Fig. 1)}

Situé au sud de la mer Tyrrhénienne et à la limite du détroit siculo-tunisien, le golfe de Tunis s'ouvre largement sur la mer par une rupture de pente atteignant plus de $100 \mathrm{~m}$. La région sud du golfe est une nouvelle entité : la baie de Tunis dont la profondeur ne dépasse pas $30 \mathrm{~m}$. La majorité des

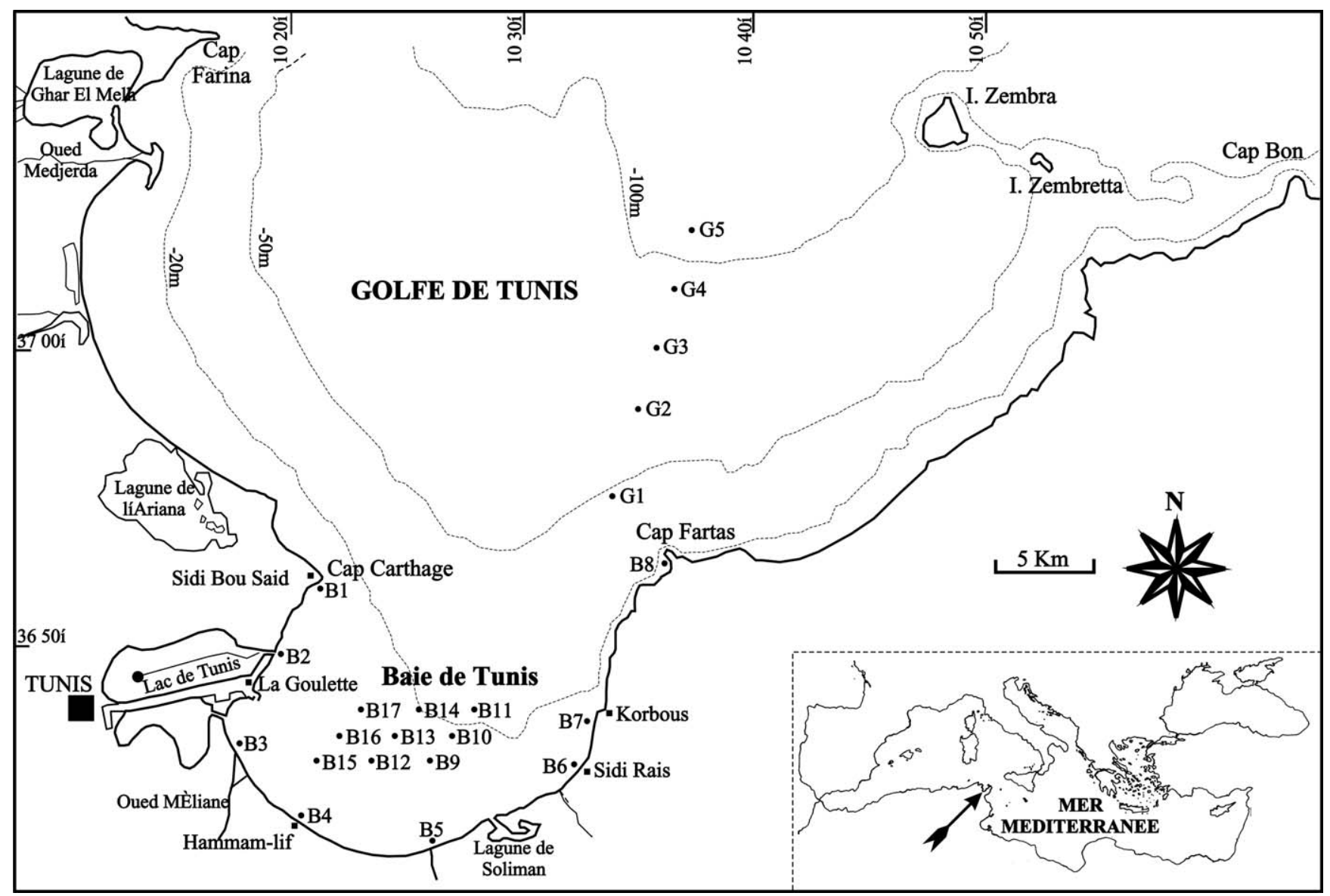

Fig. 1. Situation géographique et emplacement des stations au niveau du golfe de Tunis.

Fig. 1. Geographical position of Tunis Gulf and stations location. 
observations proviennent de la baie dont les fonds détritiques et sableux à sablovaseux sont tapissés d'un macrophytobenthos composé d'un mélange de Cymodocea nodosa et Caulerpa prolifera, tandis que les herbiers de Posidonia oceanica sont limités à la côte orientale (stations B5 à B8).

Houles, marées barométriques et courants ont, dans la baie, des effets atténués par la protection des eaux du golfe. Ces effets sont très liés aux vents dont la force et la direction peuvent inverser les courants, renouvelant ainsi la baie par des eaux d'origine atlantique ou orientale selon les vents dominants. Enfin, en raison de la faible profondeur, l'effet du vent provoque un fort mélange vertical des eaux de la baie.

\subsection{Stratégies d'échantillonnage (Fig. 1)}

Treize stations d'études ont été explorées mensuellement de décembre 1993 à novembre 1995, à l'intérieur de la baie pour l'étude du zooplancton et une radiale a prospecté les eaux du golfe, en novembre 1995, depuis les isobathes $50 \mathrm{~m}$ jusqu'à 100 m (G1 à G5).

La température et la salinité des eaux ont été mesurées in situ à l'aide d'un salinomètre de type WTW LF196 muni d'une sonde de température.

Deux filets à plancton, ayant les caractéristiques suivantes, ont été utilisés, par traits verticaux fond-surface, au niveau des stations profondes (B9 à B17 et G1 à G5) :
- un filet Nansen à fermeture de $55 \mu \mathrm{m}$ de vide de maille et $0,7 \mathrm{~m}$ de diamètre ;

- un filet Hensen-eggs de $300 \mu \mathrm{m}$ de vide de maille et $0,73 \mathrm{~m}$ de diamètre.

Pour les stations côtières, des traits obliques fond-surface ont été réalisés à l'aide d'un petit filet standard de $55 \mu \mathrm{m}$ de vide de maille : le macroplancton gélatineux (Scyphoméduses) étant compté in situ et récolté manuellement. Dans tous les cas les volumes filtrés sont mesurés à l'aide d'un volucompteur.

\section{Résultats}

La salinité des eaux, de la baie de Tunis, est caractérisée par une salinité maximale, au cours du mois de d'août (37,87 en 1994 et 37,81 en 1995), période de fortes évaporations. La salinité minimale est enregistrée en février 1994 $(37,07)$ et en octobre $1995(36,81)$ suite à d'importantes précipitations (Fig. 2a). La température des eaux varie de $13,2^{\circ} \mathrm{C}$ en janvier à $28,9^{\circ} \mathrm{C}$ en août (Fig. 2b).

Une des particularités de la baie est sa concentration en sels nutritifs relativement élevée dans sa partie ouest, due aux rejets urbains, industriels et agricoles (percolation des engrais) de la région de Tunis (Souissi et al., 2000).
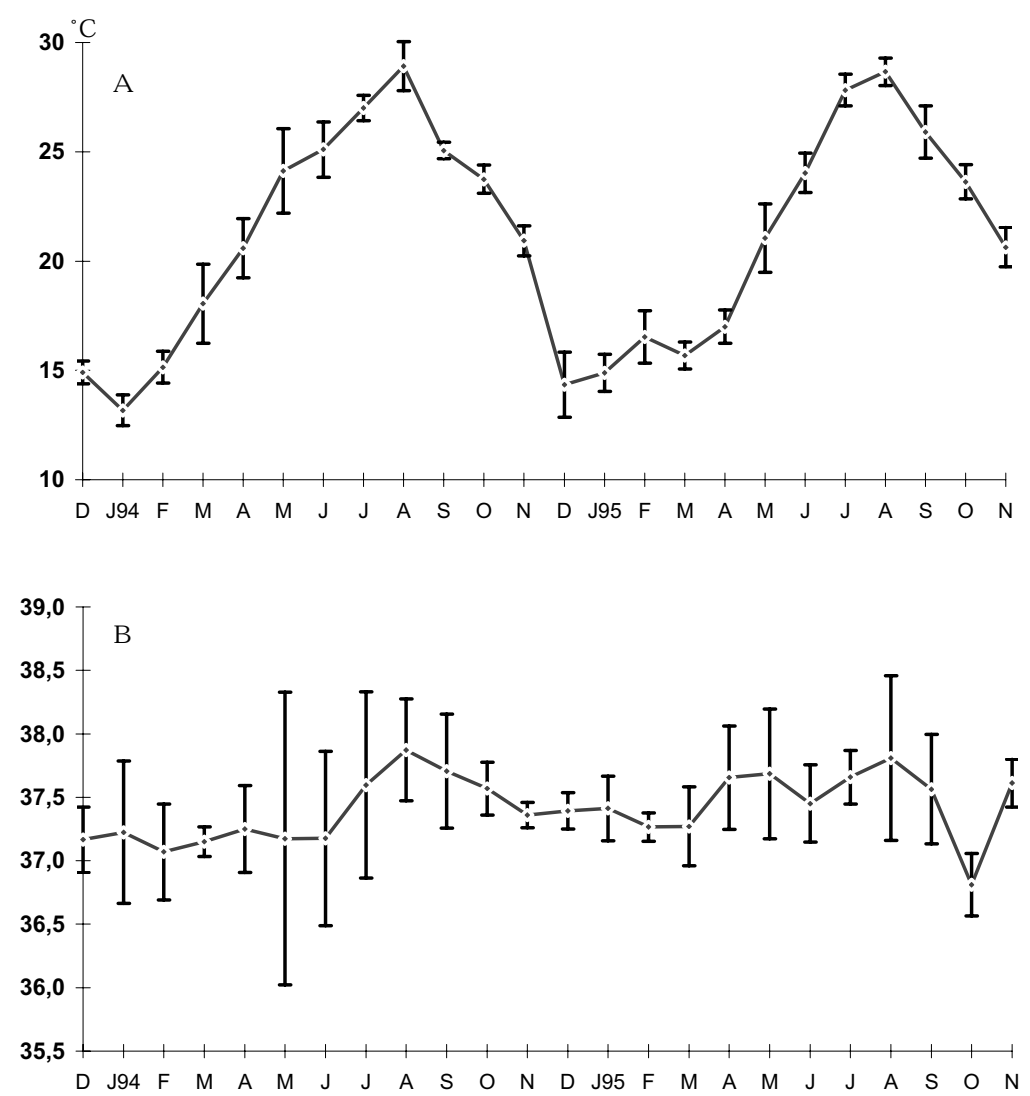

Fig. 2. Évolution saisonnière moyenne de la salinité (A) et de la température (B) au niveau de la baie de Tunis (décembre 1993-novembre 1995). Fig. 2. Temporal distribution of salinity (A) and temperature (B) in Tunis Bay between December 1993 and November 1995. 
Tableau 1

Composition taxonomique et distribution mensuelle des Méduses dans la Baie de Tunis. (* signifie rencontrée uniquement dans le golfe de Tunis au cours de la campagne (C.) du 16 au 18 novembre 1995 )

Table 1

Taxonomic composition and monthly density range of jellyfish in the Bay of Tunis. (* signify observed only in Tunis Gulf during the cruise (C.) between 16 and 18 November 1995)

\begin{tabular}{|c|c|c|c|c|c|c|c|c|c|c|c|c|c|c|c|c|c|c|c|c|c|c|c|c|c|c|c|}
\hline & Unités systématiques & D & J94 & $\mathbf{F}$ & M & A & $\mathbf{M}$ & $\mathbf{J}$ & $\mathbf{J}$ & A & $\mathrm{s}$ & o & $\mathrm{N}$ & $\mathbf{D}$ & J95 & $\mathbf{F}$ & $\mathbf{M}$ & A & M & $\mathbf{J}$ & $\mathbf{J}$ & A & s & o & $\mathrm{N}$ & $\mathrm{N}(\mathrm{C})$. & $\begin{array}{c}\text { Fréquence } \\
\text { d'occurrence }\end{array}$ \\
\hline \multirow{25}{*}{$\begin{array}{l}\mathrm{H} \\
\mathrm{Y} \\
\mathrm{D} \\
\mathrm{R} \\
\mathrm{O} \\
\mathrm{M} \\
\mathrm{E} \\
\mathrm{D} \\
\mathrm{D} \\
\mathrm{U} \\
\mathrm{S} \\
\mathrm{E} \\
\mathrm{S}\end{array}$} & Leptoméduses (4) & & & & & & & & & & & & & & & & & & & & & & & & & & \\
\hline & Obelia spp variae & & & & & & & & & & & & & & & & & & & & & & & & & & 22224 \\
\hline & Clytia spp variae & & & & & & & & & & & & & & & & & & & & & & & & & & $17 / 24$ \\
\hline & Eucheilota paradoxica Mayer, 1900 & & & & & & & & & & & & & & & & & & & & & & & & & & $5 / 24$ \\
\hline & Eutima gracilis (Forbes et Goodsir, 1853) & & & & & & & & & & & & & & & & & & & & & & & & & & $2 / 24$ \\
\hline & Anthoméduses (8) & & & & & & & & & & & & & & & & & & & & & & & & & & \\
\hline & Lizzia blondina Forbes, 1848 & & & & & & & & & & & & & & & & & & & & & & & & & & 6/24 \\
\hline & Cladonema radiatum Dujardin, 1843 & & & & & & & & & & & & & & & & & & & & & & & & & & $3 / 24$ \\
\hline & Bougainvillia ramosa (Van Beneden, 1844) & & & & & & & & & & & & & & & & & & & & & & & & & & $3 / 24$ \\
\hline & Zanclea costata Gegenbaur, 1856 & & & & & & & & & & & & & & & & & & & & & & & & & & $2 / 24$ \\
\hline & Velella velella (Linné, 1758) & & & & & & & & & & & & & & & & & & & & & & & & & & $1 / 24$ \\
\hline & Ectopleura dumortieri (Van Beneden, 1844)" & & & & & & & & & & & & & & & & & & & & & & & & & & $1 / 24$ \\
\hline & Eucodonium brownei Hartlaub, $1907^{*}$ & & & & & & & & & & & & & & & & & & & & & & & & & & $1 / 24$ \\
\hline & Podocoryne carnea M. Sars, $1846^{*}$ & & & & & & & & & & & & & & & & & & & & & & & & & & $1 / 24$ \\
\hline & Trachyméduses (5) & & & & & & & & & & & & & & & & & & & & & & & & & & \\
\hline & Sminthea eurygaster Gegenbaur, 1856 & & & & & & & & & & & & & & & & & & & & & & & & & & $8 / 24$ \\
\hline & Aglaura hemistoma Peron et Lesueur, 1810 & & & & & & & & & & & & & & & & & & & & & & & & & & 6/24 \\
\hline & Rhopalonema velatum Gegenbaur, 1856 & & & & & & & & & & & & & & & & & & & & & & & & & & 6/24 \\
\hline & Geryonia proboscidalis Forsskal, 1775 & & & & & & & & & & & & & & & & & & & & & & & & & & $1 / 24$ \\
\hline & Liriope tetraphylla (Chamisso et Eysenhardt, 1821) ${ }^{*}$ & & & & & & & & & & & & & & & & & & & & & & & & & & $1 / 24$ \\
\hline & Limnoméduses (2) & & & & & & & & & & & & & & & & & & & & & & & & & & \\
\hline & Olindias phosphorica (Delle Chiaje, 1841) & & & & & & & & & & & & & & & & & & & & & & & & & & $3 / 24$ \\
\hline & Pochella oligonema Kramp, 1955 & & & & & & & & & & & & & & & & & & & & & & & & & & $2 / 24$ \\
\hline & Narcoméduses (1) & & & & & & & & & & & & & & & & & & & & & & & & & & \\
\hline & Solmundella bitentaculata Quoy et Gaimard, 1833 & & & & & & & & & & & & & & & & & & & & & & & & & & $3 / 24$ \\
\hline \multirow{7}{*}{$\begin{array}{l}\text { S } \\
\text { C } \\
\text { Y } \\
\text { P } \\
\text { H } \\
\text { o } \\
\text { M } \\
\text { E } \\
\text { D } \\
\text { U } \\
\text { S } \\
\text { E } \\
\text { S }\end{array}$} & Semaeostomes (2) & & & & & & & & & & & & & & & & & & & & & & & & & & \\
\hline & Pelagia noctiluca (Forskal, 1775) & & & & & & & & & & & & & & & & & & & & & & & & & & $10 / 24$ \\
\hline & Aurelia aurita (Linné, 1758) & & & & & & & & & & & & & & & & & & & & & & & & & & $3 / 24$ \\
\hline & Rhizostomes (3) & & & & & & & & & & & & & & & & & & & & & & & & & & \\
\hline & Rhisostoma pulmo (Macri, 1778) & & & & & & & & & & & & & & & & & & & & & & & & & & $4 / 24$ \\
\hline & Cotylorhiza tuberculata (Macri, 1778) & & & & & & & & & & & & & & & & & & & & & & & & & & $2 / 24$ \\
\hline & Stomolophus meleagris L. Agassiz, 1862 & & & & & & & & & & & & & & & & & & & & & & & & & & $1 / 24$ \\
\hline
\end{tabular}

La classification adoptée dans cette étude est celle de Kramp (1961). Toutefois, les travaux de Bouillon et al. (1993) et Boero et al. (1996) ont aussi été utilisés.

La liste des espèces inventoriées dans le cadre de cette étude ainsi que leur fréquence d'observation sont répertoriées au Tableau 1.

\subsection{Description et écologie des Hydroméduses}

Les 20 espèces recensées dans la baie et le golfe de Tunis montrent une évolution très irrégulière d'une année à l'autre (Fig. 3a). En effet, au cours de la première année d'étude, cinq périodes d'abondance sont enregistrées (décembre 1993, février-mars, 1994, mai, juillet et septembre-octobre 1994). Durant la deuxième année d'étude, seulement trois périodes d'abondance sont enregistrées (février 1995, maijuin et septembre 1995).

\subsubsection{Leptoméduses}

3.1.1.1. Obelia spp. (Kramp, 1961 ; Vives, 1966 ; Razouls and Thiriot, 1968 ; Berhaut, 1969 ; Goy, 1972 ; Brinckmann-Voss, 1987 ; Gili et al., 1987 ; Matsakis, 1990 ; Buecher, 1996).

Dans l'impossibilité de les distinguer, les cinq espèces méditerranéennes (Boero and Bouillon, 1993) sont réunies sous la dénomination spp. Leur taille est généralement de l'ordre de $1 \mathrm{~mm}$. Fréquemment observées (fréquence d'observation mensuelle $=22 / 24$ ), elles assurent, avec les Clytia, le peuplement de base des Hydroméduses de la baie de Tunis. Elles représentent en 1995 jusqu'à 96,21 \% de ce groupe, avec des densités moyennes maximales en juin 1995 de l'ordre de $1856 \mathrm{ind} / \mathrm{m}^{3}$ (Fig. 3b). Ces densités résultent d'une poussée de $25800 \mathrm{ind} / \mathrm{m}^{3}$ à la station B2, à la sortie du lac nord de Tunis, individus qui ne s'expatrient cependant pas vers les autres stations. Dans le golfe de Tunis, Obelia spp. montre une densité moyenne faible $\left(3,2 \mathrm{ind} / \mathrm{m}^{3}\right)$ avec des valeurs ponctuelles de $14 \mathrm{ind} / \mathrm{m}^{3}$ à la station $\mathrm{G} 4$.

3.1.1.2. Clytia spp. (Kramp, 1961 ; Vives, 1966 ; Razouls et Thiriot, 1968 ; Goy, 1972 ; Brinckmann-Voss, 1987 ; Gili et al., 1987 ; Matsakis, 1990 ; Buecher, 1996).

Dans ce genre aussi, les 11 espèces méditerranéennes (Boero and Bouillon, 1993 ; Boero et al., 1996) ne peuvent être différenciées. Leur taille varie de 3 à $4 \mathrm{~mm}$. Fréquemment observées $(F=17 / 24)$, elles laissent apparaître trois périodes d'abondance : février, juillet et septembre-octobre. Les densités maximales atteignent $63 \mathrm{ind} / \mathrm{m}^{3}$ en octobre 1994 

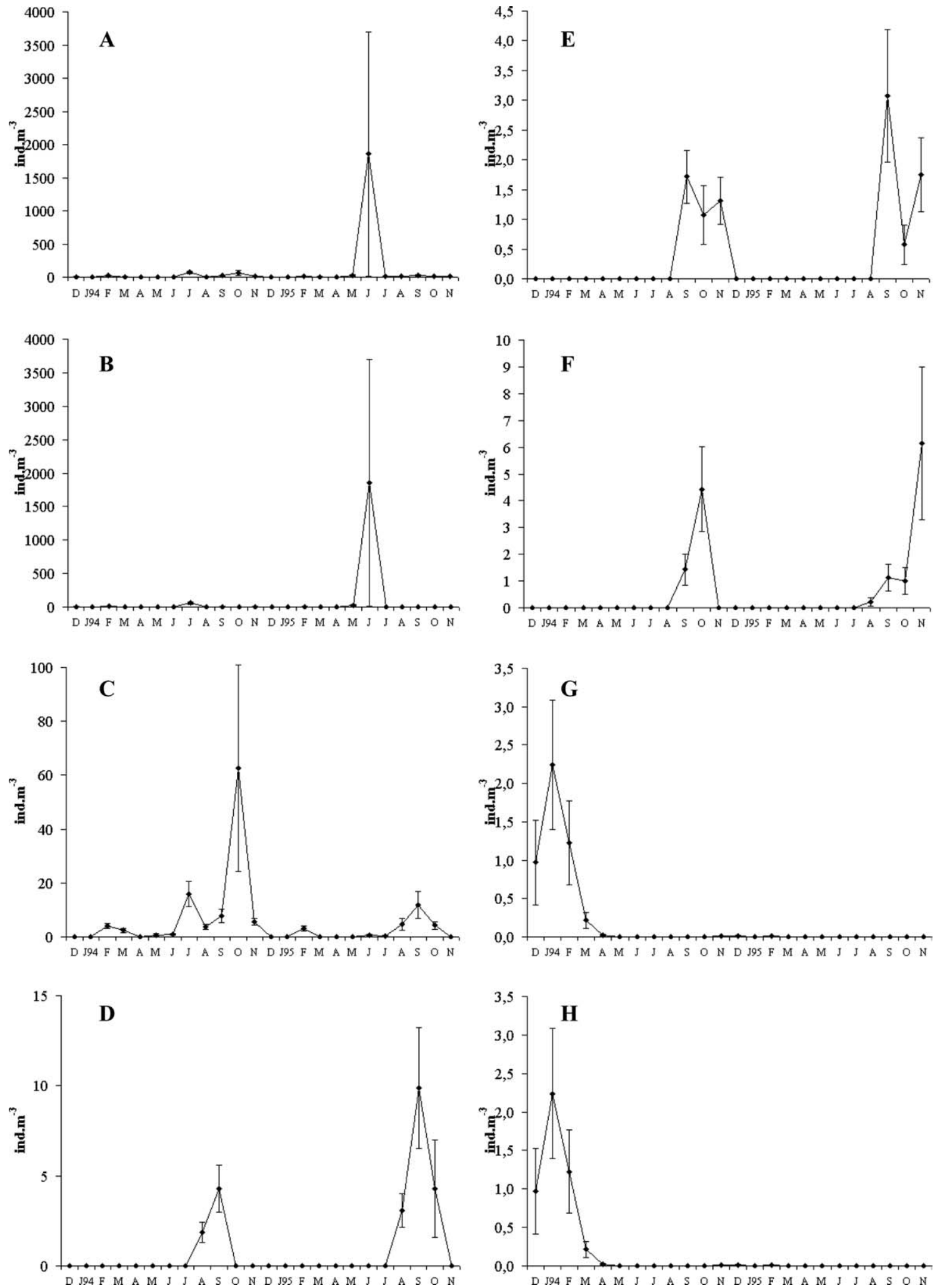

Fig. 3. (A-H). Évolution mensuelle de la densité des Hydroméduses et des Scyphoméduses ainsi que de leurs principales espèces. (A : Hydroméduses ; $\mathbf{B}:$ Obelia spp $; \mathbf{C}:$ Clytia spp $; \mathbf{D}:$ Eucheilota paradoxica $; \mathbf{E}:$ Lizzia blondina $; \mathbf{F}:$ Aglaura hemistoma $; \mathbf{G}:$ Scyphoméduses $; \mathbf{H}:$ Pelagia noctiluca). Fig. 3. (A-H). Monthly density variation of Hydromedusea, Schyphomedusea and principal jellyfish species. (A : Hydromedusea ; B : Obelia spp ; C : Clytia spp ; D : Eucheilota paradoxica ; E : Lizzia blondina ; F : Aglaura hemistoma ; G : Scyphomedusea ; H : Pelagia noctiluca). 
(Fig. 3c). Des valeurs ponctuelles extrêmes de l'ordre de 420 et $380 \mathrm{ind} / \mathrm{m}^{3}$, sont enregistrées au cours de ce même mois, respectivement, au niveau des stations B12 et B15. Dans le golfe de Tunis, ses effectifs moyens sont plus faibles $\left(24,8 \mathrm{ind} / \mathrm{m}^{3}\right)$ et atteignent $66 \mathrm{ind} / \mathrm{m}^{3}$ à la station $\mathrm{G} 4$.

Les Obelia et les Clytia quasi-pérennes dans la baie, sont considérées comme endogènes en raison de leur présence aussi bien au niveau des stations centrales, qu'au niveau des stations côtières.

3.1.1.3. Eucheilota paradoxica Mayer, 1900. (Kramp, 1961 ; Matsakis, 1990 ; Carré et Carré, 1990 ; Buecher, 1996).

De taille variant entre 2 et $3 \mathrm{~cm}$, elle a été signalée pour la première fois en Méditerranée par Schmidt et Benovic (1977), en Adriatique, et est désormais largement répandue depuis le détroit de Gibraltar jusqu' aux côtes libanaises (Goy et al., 1991). Peu fréquente $(F=5 / 24)$, elle accuse des densités moyennes voisines de $10 \mathrm{ind} / \mathrm{m}^{3}$ en septembre 1995 , associées à des blooms ponctuels de $40 \mathrm{ind} / \mathrm{m}^{3}$ enregistrés à la station B17 (Fig. 3d).

3.1.1.4. Eutima gracilis (Forbes et Goodsir, 1853). (Kramp, 1961 ; Goy, 1972 ; Brinckmann-Voss, 1987).

Sa taille dépasse $1 \mathrm{~cm}$ de diamètre et le long manubrium est recouvert par les quatre gonades. Rare au niveau de la baie de Tunis, elle n'est observée qu'à deux reprises (avril 1994 et mai 1995) avec des densités ne dépassant pas les $1 \mathrm{ind} / \mathrm{m}^{3}$.

\subsubsection{Anthoméduses}

3.1.2.1. Velella velella (Linné, 1758). (Brinckmann-Voss, 1970, 1987 ; Larson, 1980).

Les colonies d'hydraires, vivant en bancs serrés en surface au large, sont poussées en hiver dans la baie par des vents de NW dominants et persistants et s'y accumulent avant d'être rejetées au niveau des stations côtières de la région Est. Ces hydraires n'ont été observés qu'une seule fois avec des densités de l'ordre de 6 ind $/ \mathrm{m}^{3}$ au niveau de la station B2.

3.1.2.2. Zanclea costata (Gegenbaur, 1856). (Kramp, 1961 ; Berhaut, 1969 ; Goy, 1972 ; Brinckmann-Voss, 1970, 1987 ; Gili et al., 1987 ; Matsakis, 1990 ; Buecher, 1996 ; Boero et al., 2000).

De 2 à $3 \mathrm{~mm}$ de diamètre, elle n'a été observée dans la baie que de manière sporadique, en mars et octobre 1995, et se trouve le plus souvent limitée aux stations centrales avec des densités ne dépassant pas 1 ind. $/ \mathrm{m}^{3}$.

3.1.2.3. Cladonema radiatum (Dujardin, 1843). (Kramp, 1961 ; Berhaut, 1969 ; Brinckmann-Voss, 1970, 1987 ; Goy, 1972).

De 3 à $4 \mathrm{~mm}$ de hauteur, elle est associée aux herbiers de Cymodocées et de Posidonies et se développe en saison estivale avec $8 \mathrm{ind} / \mathrm{m}^{3}$ en juillet 1995 .
3.1.2.4. Bougainvillia ramosa (van Beneden, 1844). (Kramp, 1961 ; Razouls and Thiriot, 1968 ; Berhaut, 1969 ; Goy, 1972 ; Brinckmann-Voss, 1987 ; Gili et al., 1987 ; Matsakis, 1990 ; Buecher, 1996).

Très petite méduse ( $2 \mathrm{~mm}$ de hauteur), elle fait partie de la faune commune aux deux bassins. Elle est à la fois très côtière et très superficielle mais n'est jamais récoltée en grand nombre $\left(0,4 \mathrm{ind} / \mathrm{m}^{3}\right.$ en mai 1995). Ses apparitions sporadiques dans la baie se limitent à la saison printanière.

3.1.2.5. Lizzia blondina (Forbes, 1848). (Kramp, 1961 ; Razouls et Thiriot, 1968 ; Berhaut, 1969 ; Goy, 1972 ; Gili et al., 1987 ; Buecher, 1996).

Les densités moyennes de l'ordre de 3 ind $/ \mathrm{m}^{3}$, enregistrées en septembre 1995, sont l'œuvre de valeurs ponctuelles élevées atteignant $12 \mathrm{ind} / \mathrm{m}^{3}$ à la station B11 (Fig. 3e). Cette petite méduse ( 3 à $4 \mathrm{~mm}$ de hauteur) a aussi été retrouvée au niveau de la station G5 avec des densités de $2 \mathrm{ind} / \mathrm{m}^{3}$.

3.1.2.6. Ectopleura dumortieri (Van Beneden, 1844). (Kramp, 1961 ; Berhaut, 1969 ; Goy, 1972 ; Brinckmann-Voss, 1970, 1987 ; Buecher, 1996).

La présence de huit côtes de cnidocytes sur l'exombrelle permet de distinguer aisément cette espèce, de $1 \mathrm{~mm}$ de diamètre, dans les pêches. Cette espèce a été observée une seule fois, au niveau de la station G2 du golfe de Tunis, avec une abondance maximale de $2 \mathrm{ind} / \mathrm{m}^{3}$.

3.1.2.7. Eucodonium brownei (Hartlaub, 1907). (Kramp, 1961 ; Berhaut, 1969 ; Goy, 1972 ; Brinckmann-Voss, 1970, 1987 ; Buecher, 1996).

Petite méduse d'à peine $0,5 \mathrm{~mm}$ de diamètre, très colorée en brun foncé au niveau des quatre bulbes tentaculaires et de l'estomac central, elle présente de nombreux bourgeons médusaires autour du manubrium. Absente des eaux de la baie, elle n'a été recensée qu'au niveau du golfe de Tunis (stations G3 et G4). L'abondance maximale atteinte est de $20 \mathrm{ind} / \mathrm{m}^{3}$ au niveau de G4.

3.1.2.8. Podocoryne carnea (M. Sars, 1846). (Kramp, 1961 ; Vives, 1966 ; Razouls et Thiriot, 1968 ; Goy, 1972 ; Berhaut, 1969 ; Brinckmann-Voss, 1987 ; Gili et al., 1987 ; Matsakis, 1990 ; Buecher, 1996).

Uniquement deux spécimens, de 2,5 mm de diamètre, sont observés au niveau de la station G1 du golfe de Tunis.

\subsubsection{Limnoméduses}

3.1.3.1. Olindias phosphorica (Delle Chiaje, 1841). (Kramp, 1961 ; Goy, 1972 ; Brinckmann-Voss, 1987 ; Buecher, 1996).

Son apparition dans la baie a été très brève, de faible intensité avec de 1 à $2 \mathrm{ind} / \mathrm{m}^{3}$ et limitée au mois d'août et de manière moins fréquente en septembre. Sa grande taille, jusqu'à $6 \mathrm{~cm}$ de diamètre, le grand nombre de tentacules urticants jusqu'à 180, et ses invasions périodiques de la côte en été, en font une espèce redoutée des baigneurs et même 
des pêcheurs. Elle est récoltée dans la partie orientale de la baie dans le secteur envahi par les posidonies sur lesquelles cette méduse est souvent posée.

\subsubsection{Pochella oligonema (Kramp, 1955). (Kramp, 1961 ;} Goy et al., 1991).

C'est la deuxième mention de cette espèce $(1 \mathrm{~mm}$ de hauteur) en Méditerranée après sa découverte sur les côtes du Liban. Son aire de répartition est restreinte à cette mer et au très proche Atlantique (Kramp, 1961). Dans la baie, elle n'est observée qu'en saison estivale, au niveau de la région centrale nord-est ( $1 \mathrm{ind} / \mathrm{m}^{3}$ à la station B11 en août 1995) : ce qui lui confère un caractère océanique et exogène.

\subsubsection{Trachyméduses}

3.1.4.1. Geryonia proboscidalis (Forsskal, 1775). (Kramp, 1961 ; Vives, 1966 ; Goy, 1972 ; Brinckmann-Voss, 1987).

Avec un diamètre de 5 à $8 \mathrm{~cm}$, c'est une des plus grandes hydroméduses rencontrées dans la baie, où elle est extrêmement rare et exogène. Elle n'a été observée qu'une seule fois, durant le mois de septembre 1995, avec des densités de l'ordre de $0,3 \mathrm{ind} / \mathrm{m}^{3}$.

\subsubsection{Aglaura hemistoma (Péron et Lesueur, 1810).} (Kramp, 1961 ; Vives, 1966 ; Razouls et Thiriot, 1968 ; Berhaut, 1969, 1970 ; Goy, 1972 ; Brinckmann-Voss, 1987 ; Gili et al., 1987 ; Matsakis, 1990 ; Buecher, 1996).

L'hauteur de l'ombrelle varie entre 4 et $5 \mathrm{~mm}$. Cette espèce est présente dans tous les inventaires méditerranéens et vient au $4^{\mathrm{e}}$ rang dans l'abondance des méduses de la baie dans la période d'homothermie chaude d'août à novembre. Avec des densités atteignant $6 \mathrm{ind} / \mathrm{m}^{3}$ en novembre 1995 (Fig. 3f), les individus sont souvent concentrés sur les stations B10-B11, B13-B14 et B16-B17 les plus profondes : ce qui confirme son caractère océanique. Toutefois, seulement deux individus ont été observés à la station G3 dans le golfe de Tunis.

3.1.4.3. Rhopalonema velatum (Gegenbaur, 1856). (Kramp, 1961 ; Vives, 1966 ; Razouls et Thiriot, 1968 ; Berhaut, 1969, 1970 ; Goy, 1972 ; Brinckmann-Voss, 1987 ; Gili et al., 1987 ; Matsakis, 1990 ; Buecher, 1996).

Cette espèce, dont la taille est de l'ordre de $10 \mathrm{~mm}$ de diamètre, est également très répandue dans les deux bassins et son caractère océanique a toujours été mentionné, souvent en relation avec les fortes salinités (Berhaut, 1970). Cependant, dans la baie de Tunis, elle est hivernale dans des eaux voisines de 37 , avec des invasions au cours des mois de février (3,43 ind $/ \mathrm{m}^{3}$ en 1994 et $2,82 \mathrm{ind} / \mathrm{m}^{3}$ en 1995), septembre $1995\left(0,75 \mathrm{ind} / \mathrm{m}^{3}\right)$ et novembre $1995\left(1 \mathrm{ind} / \mathrm{m}^{3}\right)$. Cette espèce, qui reste le plus souvent cantonnée aux stations du large, s'observe dans le golfe en novembre 1995 $\left(0,8 \mathrm{ind} / \mathrm{m}^{3}\right)$ : ce qui confirme son caractère océanique.
3.1.4.4. Sminthea eurygaster (Gegenbaur, 1856). (Kramp, 1961 ; Vives, 1966 ; Berhaut, 1969 ; Goy, 1972 ; Gili et al., 1987).

Cette espèce présente une taille de 9 à $10 \mathrm{~mm}$ de diamètre. Sa présence, témoigne d'un mélange vertical avec remontée d'eau car elle est toujours signalée dans les peuplements en dessous de $100 \mathrm{~m}$. Dans la baie, elle s'observe de juin $\left(0,57 \mathrm{ind} / \mathrm{m}^{3}\right.$ en 1995$)$ à octobre $\left(0,50 \mathrm{ind} / \mathrm{m}^{3}\right.$ en 1995$) \mathrm{au}$ niveau des stations les plus profondes et surtout dans la région centrale Nord-est considérée comme oligotrophe. Au cours du mois de novembre, elle ne s'observe que dans le golfe de Tunis où sa densité atteint $30 \mathrm{ind} / \mathrm{m}^{3}$ au niveau de la station $\mathrm{G} 2$.

3.1.4.5. Liriope tetraphylla (Chamisso et Eysenhardt, 1821). (Kramp, 1961 ; Vives, 1966 ; Berhaut, 1968, 1969 ; Razouls and Thiriot, 1968 ; Goy, 1972 ; Brinckmann-Voss, 1987 ; Matsakis, 1990 ; Buecher, 1996 ; Buecher et al., 1997).

Cette espèce, la plus commune en Méditerranée, présente une taille variant de 9 à $10 \mathrm{~mm}$ de diamètre. Elle est citée dans tous les inventaires et semble fortement liée aux eaux oligotrophes (Goy, 1997) : ce qui confirme sa présence uniquement dans le golfe de Tunis à la station G5, où ses densités maximales atteignent $8 \mathrm{ind} / \mathrm{m}^{3}$.

\subsubsection{Narcoméduses}

3.1.5.1. Solmundella bitentaculata (Quoy et Gaimard, 1833). (Kramp, 1961 ; Vives, 1966 ; Razouls et Thiriot, 1968 ; Berhaut, 1969 ; Goy, 1972 ; Brinckmann-Voss, 1987 ; Gili et al., 1987 ; Matsakis, 1990 ; Buecher, 1996).

Cette petite Narcoméduse (2 à $3 \mathrm{~mm}$ de diamètre), reconnaissable à ses deux longs tentacules rigides et dressés audessus de l'ombrelle, est présente dans tous les océans d'un pôle à l'autre (Kramp, 1961). Elle est citée dans tous les inventaires. Eurytherme, euryhaline et très cosmopolite, sa présence semble en faire une espèce très opportuniste. Elle ne s'observe dans la baie qu' en octobre $\left(1 \mathrm{ind} / \mathrm{m}^{3}\right.$ à la station B11) et ne se maintient au cours du mois de novembre que dans le golfe de Tunis à la station G2 avec des effectifs atteignant $6 \mathrm{ind} / \mathrm{m}^{3}$. Sa présence au niveau des stations les plus profondes de la baie lui confère un caractère exogène et océanique.

Parmi les Hydroméduses, ce sont les Anthoméduses (huit espèces), les Trachyméduses (cinq espèces) et les Leptoméduses (quatre espèces) qui sont les plus diversifiées.

L'analyse de la répartition saisonnière montre que les Leptoméduses sont pratiquement pérennes et dominent le peuplement des méduses constituant parfois $100 \%$ du peuplement (Tableau 1 et Fig. 4). Les Narcoméduses et les Limnoméduses sont rares avec une préférence respectivement pour les saisons automnale et estivale. Les Anthoméduses sont également rares, leur apparition dans la baie étant estivale et automnale à l'exception de la colonie d'hydraire de Velella velella et Zanclea costata qui sont hivernales. Elles dominent parfois les méduses et représentent, en juillet 


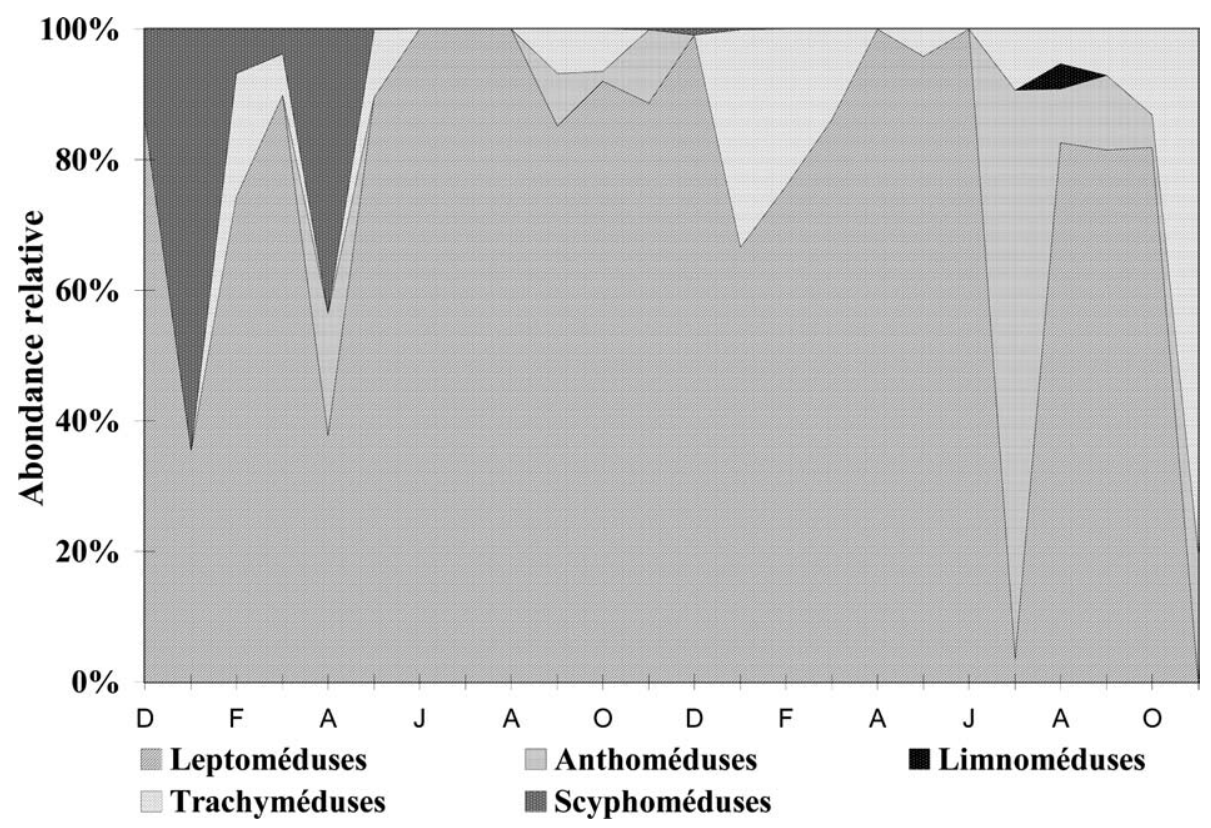

Fig. 4. Variations mensuelles de l'abondance relative des méduses de la baie de Tunis entre décembre 1993 et novembre 1995.

Fig. 4. Monthly variations of the relative abundance of Tunis Bay jellyfish between December 1993 and November 1995.

1995, plus de $80 \%$ du peuplement. Les Trachyméduses, représentées surtout par Rhopalonema velatum et Sminthea eurygaster, sont pour la plupart estivales, automnales ou hivernales (cas de $R$. velatum).

\subsection{Description et écologie des Scyphoméduses}

Les cinq espèces de Scyphoméduses recensées présentent une dynamique temporelle caractérisée par un important développement hivernal et automnal (Fig. 3g).

\subsubsection{Semaeostomes}

3.2.1.1. Pelagia noctiluca (Forsskal, 1775). (Kramp, 1961 ; UNEP, 1984 ; Gili et al., 1987 ; Morand, 1989 ; Buecher, 1996).

C'est l'espèce la plus commune parmi les Scyphoméduses de la Méditerranée, toutefois elle évite les panaches de dilution des grands fleuves, on ne la rencontre ni au large de Marseille ni au large du Nil (Goy et al., 1991). Elle a déjà été signalée comme rare sur les côtes est de la Tunisie (Hamza, 1990), dans le lac de Bizerte (Chakroun et Alaoui-Bejaoui, 1995) et au large de l'Algérie (Ranson, 1925).

Dans la baie de Tunis, la population de Pelagia noctiluca présente une taille variant de 3 à $4 \mathrm{~cm}$. Ses fluctuations mensuelles montrent que cette espèce domine le peuplement des Scyphoméduses (Fig. 3g, h) et fréquente les côtes de novembre à mai. Toutefois, d'une année à l'autre, les invasions sont variables dans le temps et selon leurs densités. C'est au cours de la première invasion (décembre 1993-mai 1994) que les densités ont été les plus importantes. Nous avons enregistré des concentrations moyennes maximales de près de $2,24 \mathrm{ind} / \mathrm{m}^{3}$ en janvier 1994 , avec des valeurs ponctuelles de près de $7,22 \mathrm{ind} / \mathrm{m}^{3}$ en février 1994 , sur la côte est de la baie de Tunis. L'étude de la direction et de la force des vents montre que ces agrégations de Pelagia noctiluca, sur le littoral Est, sont directement sous l'influence des courants induits par les vents de secteur NW et WNW très fréquents au cours de la saison hivernale.

3.2.1.2. Aurelia aurita (Linné, 1758). (Kramp, 1961 ; Morand et Dallot, 1985).

Faisant partie de tous les inventaires méditerranéens, sa taille dans la baie de Tunis se situe entre 6 et $8 \mathrm{~cm}$ de diamètre. Elle n'a été observée qu'au cours des mois de mai 1994 et juin 1994-1995 et ses densités moyennes maximales ne dépassent pas $0,3 \mathrm{ind} / \mathrm{m}^{3}$.

\subsubsection{Rhizostomes}

3.2.2.1. Cotylorhiza tuberculata (Macri, 1778). (Kramp, 1961 ; Morand et Dallot, 1985 ; Buecher, 1996).

Grosse Scyphoméduse, atteignant $20 \mathrm{~cm}$ de diamètre, elle est considérée comme endémique de la Méditerranée (Kramp, 1961), toutefois deux observations à la fin du siècle dernier en mer Rouge et aux Canaries semblent indiquer une lente progression de son aire de répartition (Mayer, 1910).

Pour Morand et Dallot (1985), c'est une espèce estivale dans le macroplancton de la mer Ligure. Dans la baie de Tunis, elle est très rare et son apparition semble limitée au mois d'octobre et parfois novembre.

\subsubsection{Rhizostoma pulmo (Macri, 1778). (Kramp, 1961 ;} Morand et Dallot, 1985 ; Buecher, 1996).

C'est également une espèce endémique de la Méditerranée qui se différencie de l'espèce atlantique $R$. octopus par un diamètre plus faible et un nombre réduit de lobes ombrel- 
laires, caractères que Russell (1970) estime suffisamment constants pour distinguer les deux espèces en suivant les observations de Péron et Lesueur (1810). À l'inverse de l'espèce précédente, c'est une espèce « régulière » dans la faune pélagique qui présente une forte dépression estivale dans ses effectifs (Morand et Dallot, 1985). Dans la baie de Tunis, sa taille varie entre 20 et $40 \mathrm{~cm}$. Sa période de présence semble être les mois de mai, de septembre et d'octobre durant lesquels des densités maximales de $0,003 \mathrm{ind} / \mathrm{m}^{3}$ ont été observées au niveau de la station B12.

\subsubsection{Stomolophus meleagris (L. Agassiz, 1862). (Kramp,} 1961 ; Morand et Dallot, 1985).

Connue des zones chaudes de l'océan Pacifique et de l'océan Atlantique, la récolte d'un exemplaire très jeune (1 cm de diamètre) à la station B2 en mai 1995 étend l'aire de répartition de cette espèce à la Méditerranée. Comme l'adulte peut atteindre $20 \mathrm{~cm}$ de diamètre, sa présence ne peut passer inaperçue surtout avec le quadrillage serré des stations opéré depuis 1993.

\section{Conclusion}

Dans la baie de Tunis les méduses constituent du point de vue qualitatif, avec 25 espèces recensées, le troisième groupe après les Tintinnides et les Copépodes pélagiques (Daly Yahia et al., 2001). Les Hydroméduses (Fig. 3a) montrent que les densités moyennes les plus fortes sont enregistrées en juin $1995\left(1857 \mathrm{ind} / \mathrm{m}^{3}\right)$. Les autres périodes d'abondance s'observent au cours de l'année en février et septembreoctobre, mais les densités moyennes sont beaucoup plus faibles, de l'ordre de 15 à $30 \mathrm{ind} / \mathrm{m}^{3}$. Ce sont les espèces du genre Obelia qui commandent la dynamique de l'ensemble des Hydroméduses. L'évolution mensuelle des Obelia spp. laisse apparaître au cours de la première année de l'étude cinq périodes distinctes. La première émission est hivernale en février-mars, la deuxième est printanière en mai-juin, la troisième est estivale en juillet, la quatrième et la cinquième s'observent en automne (septembre et novembre). Ces différentes périodes d'émissions dans l'année résulteraient selon Russell (1953) d'espèces d'Obelia distinctes. En effet, cet auteur montre que les Hydraires émettent une seule fois leurs méduses par an. Ainsi, l'existence de cinq espèces méditerranéennes décrites par Boero et Fresi (1986) et Boero et Bouillon (1993) est confirmée dans ce travail. On peut de la même façon considérer que les trois pics de Clytia spp. observés en février, juillet et septembre-octobre correspondent à la présence de trois des 11 espèces méditerranéennes existantes dans la baie (Fig. 3c). Une situation identique est signalée en mer Ligure (Goy, 1972). Depuis les travaux de Elmhirst (1925), le synchronisme entre les libérations de méduses et la pleine lune est observé et, effectivement sur les 24 mois d'étude, la majorité des pics de densité est enregistrée durant les périodes de pleine lune.

Il semble que le lac nord de Tunis, (après son assainissement et son aménagement réalisé en 1988) soit très favorable, en raison de sa riche végétation macrobenthique, à l'installation d'hydraires d'Obelia spp. Il serait ainsi intéressant d'étudier les périodes où la phase leptoméduse libre apparaît. En effet, ces essaims de méduses doivent par leur prédation avoir un impact important sur les populations microzooplanctoniques et mésozooplanctoniques en place.

Les Scyphoméduses sont, quant à elles, représentées par cinq espèces, Pelagia noctiluca, la plus commune, est automnale et hivernale, disparaissant au milieu du printemps. Si sur les côtes françaises, italiennes et espagnoles, Pelagia noctiluca a pullulé au cours de la saison estivale de 1984 à 1987 (Bernard et al., 1988), sur le littoral tunisien, il semblerait qu'elle disparaisse de la zone néritique dès le mois de mars et exceptionnellement à partir du mois de mai. De plus, sur les côtes méditerranéennes nord occidentales, les invasions en masse des adultes sur les plages s'observent en saisons estivale et automnale, alors que ce phénomène a lieu surtout à la saison hivernale sur les côtes tunisiennes.

Aurelia aurita semble surtout liée aux eaux eutrophisées et dessalées. Cependant, si on ne la rencontre pas sur les côtes libanaises (Goy et al., 1991), elle est bien représentée dans le lac de Bizerte (Chakroun et Alaoui-Bejaoui 1995) et en mer Egée (Kiortsis, 1969 ; UNEP, 1984, 1991). Toutefois, ces zones sont aussi des portions à salinités relativement faibles en Méditerranée, proches de celles des mers plus boréales où l'espèce est très abondante : c'est ce qui expliquerait son absence des eaux les plus salées de la Méditerranée, proches de 39, comme les eaux libanaises ou encore sur le littoral Est de la Tunisie (ex. Baie de Sousse).

Parmi les Scyphoméduses, une attention particulière doit être donnée à Stomolophus meleagris. L'existence de ports internationaux dans la baie de Tunis (ports de Tunis, de La Goulette et de Radès), ainsi que l'absence de l'adulte, seraient en faveur de l'hypothèse d'une introduction de l'espèce par les eaux de déballastage des navires plutôt que l'existence d'une relique. De plus Boudouresque et Ribera (1994) montrent que ce déballastage des navires constitue le principal vecteur d'introduction d'espèces, particulièrement les espèces planctoniques. Selon ces mêmes auteurs le temps de survie des organismes dans les eaux de ballast peut dépasser 18 jours, et le nombre d'espèces que certains navires sont susceptibles de transporter peut atteindre le nombre de 400 .

Dans le golfe de Tunis cohabitent des espèces méroplanctoniques qui colonisent rapidement les substrats et sont présentes dans toute la Méditerranée : il s'agit par exemple d'Obelia et de Clytia ou encore Cladonema radiatum et Olindias phosphorica espèces vivant sur les herbiers de posidonies. On rencontre aussi un lot d'espèces holoplanctoniques comme Aglaura hemistoma, Rhopalonema velatum et Solmnundella bitentaculata présentes aussi bien dans les eaux du large que dans les eaux côtières. Enfin, certaines espèces à haut pouvoir bourgeonnant Lizzia blondina et Eucheilota paradoxica prennent temporairement un caractère holoplanctonique, avec une reproduction asexuée sans stade fixé, et peuvent ainsi envahir la baie très rapidement. Leur caractère océanique se confirme par leur présence au 
niveau des stations les plus au large, d'août à octobre pour Eucheilota paradoxica et de septembre à novembre pour Lizzia blondina.

Dans les eaux peu profondes du golfe de Tunis, la configuration de la côte largement ouverte permet aux eaux du large de pénétrer par vent de NE, N et NW et contribue ainsi à la présence d'espèces plus océaniques dans ce secteur. C'est ainsi que l'on peut expliquer les invasions de Pelagia noctiluca. En revanche, la présence de Sminthea eurygaster, qui est normalement mésopélagique, traduit une remontée d'eau profonde sans doute par mélange vertical ou sous l'effet d'upwellings locaux lorsque des vents de terre (secteur SW à SE) soufflent durant plusieurs jours.

Cet inventaire, réalisé pendant deux ans, confirme la pauvreté en méduses (20 Hydroméduses et cinq Scyphoméduses) des côtes méridionales de la Méditerranée déjà signalée, entre autres, par Soenen (1969), Séguin (1973) et Mills et al. (1996) qui ont recensé respectivement 22 (20 hydroméduses et deux scyphoméduses), six (cinq hydroméduses et un scyphoméduse) et 18 espèces (12 hydroméduses et six scyphoméduses).

Ainsi, si l'origine atlantique de cette faune est évidente dans les temps géologiques grâce aux grandes transgressions cénomaniennes, actuellement le jet atlantique n'apporte aucun élément qui puisse l'enrichir. Si la faune atlantique était ainsi transportée, le nombre d'espèces devrait être au moins égal à celui observé dans la partie septentrionale, or il atteint à peine le tiers des espèces recensées en mer Ligure (Goy, 1972) et un peu plus du dixième des méduses connues en Méditerranée (Kramp, 1959).

Il faut plutôt considérer le golfe de Tunis peuplé par des espèces bien implantées dans ce biotope particulier (les Hydroméduses : Obelia spp., Clytia spp., Olindias, Cladonema et les Scyphoméduses Aurelia, Cotylorhiza, Rhizostoma) avec des incursions d'espèces visiteuses (Velella, Sminthea, Pelagia) poussées vers la côte par les vents et les courants induits et qui persistent un certain temps piégées par la configuration du golfe et de la baie de Tunis beaucoup plus confinée.

Cette structure du peuplement des méduses rappelle étroitement la dynamique des Copépodes pélagiques de la baie de Tunis dont le peuplement de base est constitué d'espèces endogènes caractérisant une communauté néritique régulièrement soumises à la pulsion d'espèces exogènes ayant une origine atlantique ou océanique parfois même profonde qui pénètrent dans la baie sous l'effet de conditions climatologiques et hydrologiques particulières (Daly Yahia et al., en révision).

Il apparaît ainsi que la communauté de méduses endogènes, à la baie, présente généralement une répartition centrale sous l'influence prononcée de la région côtière ouest de la baie et en particulier de la station B2 qui communique avec le «Lac Nord » de Tunis. Tandis que la communauté exogène, d'origine océanique, caractérise essentiellement la région centrale nord-est (stations B10, B11 et B14).
Ainsi la particularité de l'écosystème planctonique que constitue le golfe de Tunis et plus précisément la baie de Tunis, réside dans la coexistence de ces deux types de communautés : l'une endogène, constante plus ou moins pérenne, l'autre exogène, à répartition spatiotemporelle très irrégulière.

\section{Remerciements}

Les auteurs remercient Mr le professeur Paul Nival pour son aide et ses conseils tout au long de la période d'étude. Ce travail est une contribution au projet CMCU (Code 00/F0912). Nous remercions également les arbitres pour leurs commentaires et suggestions.

\section{Références}

Berhaut, J., 1968. Variations mensuelles d'abondance de la Trachyméduse Liriope tetraphylla (Chamisso et Eysenhard, 1821) dans le Golfe de Marseille. Bull. Mus. Nat. Hist. Nat. Paris 40 (6), 1222-1231 2érie.

Berhaut, J., 1969. Étude qualitative, Quantitative et écologique des Hydroméduses du golfe de Marseille. Thetys 1 (3), 667-708.

Berhaut, J., 1970. Étude qualitative, quantitative et écologique des Hydroméduses du golfe de Marseille. Téthys 1 (3), 667-708.

Bernard, P., Couasnon, F., Soubiran, J.P., Goujon, J.F., 1988. Surveillance estivale de la méduse Pelagia noctiluca (Cnidaria, Scyphozoa) sur les côtes méditerranéennes françaises. Ann. Inst. Océanogr., Paris 64 (2), 115-125.

Boero, F., Bouillon, J., 1993. Zoogeography and Life Cycle Patterns on Mediterranean Hydromedusae (Cnidaria). Biol. J. Linnean Soc. 48, 239-266.

Boero, F., Fresi, E., 1986. Zonation and Evolution of a Rocky Bottom Hydroid Community. Mar. Ecol. 7, 123-150.

Boero, F., Bouillon, J., Piraino, S., 1996. Classification and phylogeny in the Hydroidomedusae (Hydrozoa, Cnidaria). Sci. Mar. 60 (1), 1-33.

Boero, F., Bouillon, J., Gravili, C., 2000. A survey of Zanclea, Halocoryne and Zanclella (Cnidaria, Hydrozoa, Anthomedusae, Zancleidae) with description of new species. Ital. J. Zool. 67, 93-124.

Boudouresque, C.F., Ribera, M.A., 1994. Les introductions d'espèces végétales et animales en milieu marin — conséquences écologiques et économiques et problèmes législatifs. First International Workshop on Caulerpa taxifolia, France.

Bouillon, J., Carré, D., Franc, A., Goy, J., Hernandez-Nicaise, M.L., Tiffon, Y., et al., 1993. Traité de Zoologie. Cnidaires - Cténaires. Anatomie, Systématique, Biologie, Tome III (2). Éditions Masson, Paris 1117 p.

Brinckmann-Voss, A., 1970. Anthomedusae-Athecatae (Hydrozoa, Cnidaria) of the Mediterranean. Part I Capitata. Fauna e Flora del Golfo di Napoli 39, 1-96.

Brinckmann-Voss, A., 1987. Seasonal distribution of Hydromedusae (Cnidaria, Hydrozoa) from the gulf of Naples and vicinity, with observations on sexual and asexual reproduction in somes species. In: Bouillon, J., Boero, F., Cicogna, F., Cornelius, P. (Eds.), Modern trends in the systematics, Ecology and Evolution of Hydroids and Hydromedusae. Oxford Science Publications, pp. 133-141.

Buecher, E., 1996. Étude écologique des carnivores gélatineux — Hydroméduses, Siphonophores, Scyphoméduses et Cténaires — dans la rade de Villefranche-sur-mer Thèse de Doctorat. Paris VI 183 p.

Buecher, E., Goy, J., Planque, B., Dallot, S., 1997. Long-term fluctuations of Liriope tetraphylla in Villefranche Bay between 1966 and 1993 compared to Pelagia noctiluca pullulations. Oceanol. Acta 20 (1), 145-157. 
Carré, D., Carré, C., 1990. Complex Reproductive Cycle in Eucheilota paradoxica (Hydrozoa: Leptomedusae): Medusae, Polyps and Frustules Produced from Medusa Stage. Mar. Biol. Berl. 104, 303-310.

Chakroun, F., Alaoui-Bejaoui, N., 1995. Invasion d'Aurelia aurita (Cnidaria, Scyphomedusa) dans le lac de Bizerte (Tunisie) au cours de l'été 1994. Ann. Inst. Océanogr. Paris 71 (1), 11-13.

CIESM, 2001. Gelatinous zooplankton outbreaks: theory and practice CIESM Workshop Series $14110 \mathrm{p}$.

Daly Yahia, M.N., Daly Yahia-Kéfi, O., Souissi, S., Romdhane, M.S., 2001. Variations spatiotemporelles du zooplancton de la baie de Tunis : composition numérique et biomasse. Rapp. Comm. int. Mer Médit. 36.

Daly Yahia M.N., Souissi S., Daly Yahia-Kéfi O., en révision. Spatiotemporal structure of planktonic copepods in the bay of Tunis (South Western Mediterranean Sea). Zool. Stud.

Elmhirst, R., 1925. Lunar periodicity in Obelia. Nature 116 (2914), 358359.

Gili, J.M., Pages, F., Vives, F., 1987. Distribution and ecology of a population of planktonic Cnidarians in the western Mediterranean. In: Bouillon, J., Boero, F., Cicogna, F., Cornelius, P. (Eds.), Modern trends in the systematics, Ecology and Evolution of Hydroids and Hydromedusae. Oxford Science Publications, pp. 157-170.

Goy, J., 1972. Les Hydroméduses de la mer Ligure. Bull. Mus. Nat. Hist. Nat. 62 (83), 965-1008 3 $3^{\mathrm{e}}$ série.

Goy, J., 1997. The Medusae (Cnidaria, Hydrozoa) and their trophic environment: an example in the north-Western Mediterranean. Annales de l'Institut Océanographique 73 (2), 159-171.

Goy, J., Lakkis, S., Zeidane, R., 1991. Les Méduses (Cnidaria) des eaux Libanaises. Ann. Inst. océanogr., Paris 67 (2), 99-128.

Hamza, A., 1990. Sur la prolifération des méduses sur certaines côtes Tunisiennes. Rapp. Doc. Inst. Nat. Sc. Tech. Océanogr. Pêche Salambô 3, 1-9.

Kiortsis, V., 1969. Marine fauna of the Aegean Sea. Surface zooplankton from the north Aegean Sea. Zoological laboratory and museum. University of Athens - Greece.

Kramp, P.L., 1959. The hydromedusae of the Atlantic Ocean and adjacent waters. "Dana" Report, 46, pp. 1-283.

Kramp, P.L., 1961. Synopsis of the Medusae of the world. J. Mar. Biol. Assoc. U.K. 40, 1-469.

Larson, R.J., 1980. The medusa of Velella velella (Linnaeus, 1758) (Hydrozoa, Chondrophorae). J. Plankton Res. 2, 183-186.

Mayer, A.G., 1910. Medusae of the world. The Hydromedusae. Publs Carnegie Inst., Washington.

Matsakis, S., 1990. Comportement et métabolisme de quelques méduses en mer Ligure et dans le bassin de Bedford Thèse de Doctorat. Paris VI $163 \mathrm{p}$.
Mills, C.E., Pugh, P.R., Harbison, G.R., Haddock, S.H.D., 1996. Medusae, siphonophores and ctenophores of the Alboran Sea, south western Mediterranean. Sci. Mar. 60 (1), 145-163.

Morand, P., 1989. La méduse Pelagia noctiluca en Méditerranée occidentale : de la dynamique de population aux variations à long terme. Doctorat de l'Université Paris-VII. Biomathématiques, 191.

Morand, P., Dallot, S., 1985. Variations annuelles et pluriannuelles de quelques espèces du macroplancton côtier de la mer Ligure (18981914). Rapp. Comm. Int. Mer Médit. 29 (9), 295-297.

Péron, F., Lesueur, C.A., 1810. Tableau des caractères génériques et spécifiques de toutes les de méduses connues jusqu'à ce jour. Ann. Mus. Hist. Nat. Paris 14, 325-366.

Ranson, G., 1925. Quelques observations sur le plankton et liste des méduses recueillies par «la tanche » pendant la croisière de 1924. Bull. Mus. Nat. Hist. Nat. 31, 379-382.

Razouls, S., Thiriot, A., 1968. Le macroplancton de la région de Banyulssur-mer (golfe du Lion). Vie Milieu 19 (1B), 133-184.

Russel, F.S., 1953. The Medusae of the British Isles. Cambridge University press.

Russell, F.S., 1970. The Medusae of the British Isles. II. Pelagic Scyphozoa with a supplement to the first volume on Hydromedusae. University press, Cambridge.

Schmidt, H.E., Benovic, A., 1977. Notes on hydromedusae (Cnidaria) from the Adriatic sea. J. Mar. Biol. Ass. UK 57, 635-640.

Séguin, G., 1968. Le plancton de la côte Nord de la Tunisie (Note préliminaire). Pelagos- Bull. Inst. Océanogr. Alger 7, 73-83.

Séguin, G., 1973. Cycles comparés du zooplancton dans l'Ouest Africain et la Méditerranée sud occidentale. Université des Sciences et Techniques de Lille Thèse de Doctorat Es Sciences Naturelles.

Soenen, M., 1969. Contribution à l'étude du Zooplancton superficiel et profond du bassin sud-occidental méditerranéen. Faculté des Sciences de l'Université d'Aix-Marseille Thèse de Doctorat de $3^{\mathrm{e}}$ cycle en Océanographie Biologique 234.

Souissi, S., Daly Yahia-Kéfi, O., Daly Yahia, M.N., 2000. Spatial characterisation of nutrient dynamics in the Bay of Tunis (south-western Mediterranean) using multivariate analyses: consequences for phyto and zooplankton distribution. J. Plankton Res. 22 (11), 2039-2059.

Trégouboff, G., Rose, M., 1957. Manuel de planctonologie méditerranéenne Tome I (texte) et tome II (planches) 587 p (207 pl).

UNEP, 1984. Workshop on jellyfish blooms in the Mediterranean Athens, 31st October-4 November 1983.

UNEP, 1991. Workshop on jellyfish blooms in the Mediterranean. MAP Tech. Rep. Ser. No 47 Trieste 2-5 September 1987.

Vives, F., 1966. Zooplancton neritico de las aguas de Castellon (Mediterraneo occidental). Inv. Pesq. 30, 49-166. 